\title{
Empathie: nicht zu viel und nicht zu wenig
}

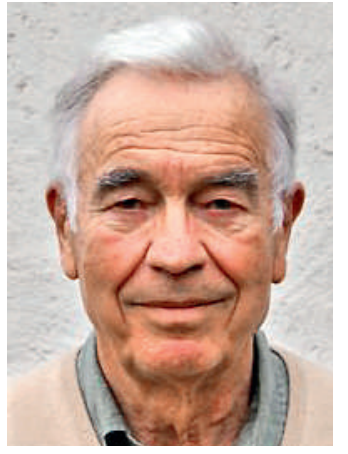

Hans Stalder

Lesen Sie hierzu auch den Beitrag von P. Weibel auf Seite 1311.

\footnotetext{
* Prof. Dr. med. Hans Stalder, Facharzt für Innere Medizin, Redaktionsmitglied, ist ehemaliger Direktor der Policlinique de Médecine und des Départements de Médecine communautaire des Hôpitaux Universitaires de Genève.
}

hans.stalder [at]saez.ch
We do not know our own souls, let alone the souls of others ... Always to have sympathy, always to be accompanied, always to be understood would be intolerable.

(Virginia Woolf: On being ill)

Empathie ist «die Bereitschaft und Fähigkeit, sich in die Einstellungen anderer Menschen einzufühlen» (Duden). Diese Eigenschaft, die uns befähigt, menschlich zu sein, wird in letzter Zeit vor allem von den Neurobiologen thematisiert, die nach der Entdeckung der Spiegelneuronen begonnen haben, die verschiedenen, der Empathie zugeordneten Strukturen und Bahnen im Gehirn zu untersuchen. Diese sowohl emotionalen als auch kognitiven Komponenten entwickeln sich sehr früh im Kleinkindalter, bereits vor Ablauf eines Jahres. Dabei gibt es genetische und geschlechtsspezifische Unterschiede. Da unser Gehirn einen gewissen Grad an Plastizität bewahrt, ist es nicht verwunderlich, dass die Fähigkeit, Empathie zu empfinden, entwickelt und somit auch vermittelt, schlimmstenfalls aber auch manipuliert und sogar unterdrückt werden kann. Empathie lässt sich mit Hilfe psychologischer Tests messen.

Empathie ist unabdingbar für jeden Arzt. Die Frage ist jedoch, wie viel Empathie es braucht, um ein guter Arzt zu sein. Verhält es sich dabei wie mit den Medikamenten: Wenn die Dosis zu schwach ist, wirken sie nicht, und ist sie zu stark, gibt es zu viele unerwünschte Nebenwirkungen? Bislang wurde in der medizinischen Literatur häufig nur der kognitive Aspekt der Empathie betont. Für eine gute Betreuung sei es zwar wichtig, die Gefühle des Patienten zu erkennen und wahrzunehmen, gleichzeitig sollte man sich jedoch auch vor einem Zuviel an Emotionen schützen, um die eigene Urteilsfähigkeit nicht zu schmälern, um die richtige Diagnose zu stellen, die richtigen Untersuchungen einzuleiten und um richtig behandeln zu können. Sich emotional distanzieren war dies nicht bereits die Devise unserer Anatomielehrer im Seziersaal? Es ist im Übrigen eine bekannte Tatsache, dass Medizinstudenten im ersten Jahr deutlich empathischer sind als am Ende ihres Studiums ...

Echte Empathie bedeutet jedoch nicht nur, die Emotionen anderer zu registrieren und zu verstehen, sondern auch deren Nachempfinden. So lässt sich beispielsweise die Angst einer Patientin vor einem Eingriff - selbst wenn sie mental registriert wird - nicht allein durch die blosse Erklärung, dass die Anästhesie jeden Schmerz nimmt oder die Statistik nur eine geringe Sterblichkeitsziffer ausweist, beschwichtigen. Angst muss nachempfunden werden, um sie besser verstehen zu können. Das Sicheinfühlen in die Emotionen anderer muss jedoch nicht zwingend zu Handlungen führen. In einer Zeit, in der überall aktives Handeln gefragt ist, haben wohl auch wir nur allzu oft die Fähigkeit des einfachen Zuhörens verloren.

\section{Wir alle haben in gewissen Momenten unter einem Zuviel an Empathie gelitten.}

Gibt es ebenfalls ein Zuviel an Empathie? Einige nennen dies Sympathie (mitleiden - der Begriff Sympathie ist natürlich noch mit anderen Bedeutungen belegt). Zu viel Empathie, oder gar unkontrollierte Empathie, kann dazu führen, dass wir uns die Emotionen des Patienten aneignen und uns mit ihm identifizieren. Das ist gefährlich für den Arzt, der damit riskiert, sich zu widersinnigen Handlungen hinreissen zu lassen. Es ist jedoch auch abträglich für den Patienten. Immer vom anderen begleitet und verstanden zu werden, kann - so Virginia Woolf - unerträglich sein und als Gewalt empfunden werden. Wir alle haben schon aus unterschiedlichsten Gründen vielleicht gelegentlich auch aus Schuldgefühlen - an zu viel Empathie gelitten: Momente, in denen uns die Not des anderen unerträglich erscheint, und wir die eigene Hilflosigkeit nicht ertragen können.

Für jeden Patienten und für jede Situation das richtige Mass an Empathie zu finden, ist wie eine Gratwanderung [1]: Für eine gute Beziehung zum Patienten ist ein Zuviel ebenso fatal wie ein Zuwenig.

Hans Stalder*

\section{Literatur}

1 Newton BW. Walking a fine line: is it possible to remain an empathic physician and have a hardened heart? Front Hum Neurosci. 2013; 7:233. doi: 10.3389/ fnhum.2013.00233. 